Visual Neuroscience (2001), 18, 1003-1010. Printed in the USA.

Copyright (C) 2001 Cambridge University Press 0952-5238/01 \$12.50

DOI: 10.1017.S0952523801186177

\title{
Processing in the probed-sinewave paradigm is likely retinal
}

\author{
S. SABINA WOLFSON AND NORMA GRAHAM \\ Department of Psychology, Columbia University, New York, New York \\ (Received March 2, 2001; ACCEPTED November 20, 2001)
}

\begin{abstract}
In the probed-sinewave paradigm—used to study the dynamics of light adaptation—a small probe of light is superimposed on a sinusoidally flickering background. Detection threshold for the probe is measured at various times with respect to the flickering background. Here we present such stimuli using three methods: monoptic (the probe and the flickering background are presented to the same eye), dichoptic (the probe is presented to one eye and the flickering background is presented to the other eye), and binocular (the probe and the flickering background are both presented to both eyes). The results suggest that the processing associated with detecting the probe is primarily in the retina (or any place with monocular input). However, the results also suggest a slight amount of processing in the cortex (or any place with binocular input), particularly at the higher frequency of flickering background used here $(9.4 \mathrm{~Hz} v s .1 .2 \mathrm{~Hz})$. A simple schematic model with three ocular-dominance channels is consistent with the results.
\end{abstract}

Keywords: Probed-sinewave paradigm, Temporal adaptation, Light adaptation, Dichoptic, Monoptic

\section{Introduction}

The probed-sinewave paradigm has been used to explore the dynamics of light adaptation and has been found to be a strong test of light-adaptation models (Hood et al., 1997). Results from the probed-sinewave paradigm have been compared to various models' predictions with varying degrees of success. The model of Snippe et al. (2000) has been compared to probed-sinewave data in that paper and in Wolfson and Graham (2001). The model of Wilson (1997) has been compared to probed-sinewave data in Hood and Graham (1998), DeMarco et al. (2000), Shady (1999), and Wolfson and Graham (2000, 2001). Such comparisons are useful in evaluating a model, but it is also useful to consider the biological plausibility of the processes within a model. The first step is to determine whether the biological substrates for this task are primarily retinal or cortical. The experiments reported here suggest that the majority of the processing involved in the probedsinewave task is likely retinal.

In the probed-sinewave paradigm, threshold is measured for detecting a brief test probe superimposed on a temporally modulating background (for example, Fig. 1). Detection threshold for the test probe is measured at various times with respect to the modulating background (for example, Fig. 2).

This type of experiment was introduced by Boynton et al. (1961) using squarewave modulation of the background. Subsequent early work by Shickman (1970) and Maruyama and Takahashi (1977) used sinusoidal modulation (as we do here). The

Address correspondence and reprint requests to: S. Sabina Wolfson, Department of Psychology, Columbia University, New York, NY 10027, USA. E-mail: sabina@psych.columbia.edu probed-sinewave paradigm combines pieces from two different traditions of studying light adaptation: the periodic tradition, in which temporal contrast sensitivity for a flickering light is measured (e.g. de Lange, 1958; Kelly, 1961), and the aperiodic tradition, in which, for example, threshold is measured for a small, brief probe presented on a large, longer flash (e.g. Crawford, 1947; Geisler, 1978; Hood, 1978; Adelson, 1982). For excellent reviews of the light-adaptation literature, see Shapley and Enroth-Cugell (1984), Hood and Finkelstein (1986), and Hood (1998). Graham and Hood (1992; von Wiegand et al., 1995) constructed merged models which could account for results from both the periodic and aperiodic traditions (these merged models combined pieces of individual models which could account for results from the periodic or the aperiodic tradition, but not from both traditions). However, Hood et al. (1997) found that these merged models could not account for important characteristics of probed-sinewave data. Subsequently, the models of Wilson (1997) and Snippe et al. (2000) were introduced and compared to probed-sinewave data. These two models have been relatively successful.

Some probed-sinewave results are shown in Fig. 3. Detection threshold for decrement probes was measured at eight different phases with respect to the flicking background (spatial configuration in Fig. 1, temporal characteristics in Fig. 2, and procedure as in Wolfson \& Graham, 2000). As the frequency of the flickering background increased (from $1.2 \mathrm{~Hz}$ to $9.4 \mathrm{~Hz}$ in Fig. 3), the overall height of the curve (the average threshold elevation, also called the dc-level) increased markedly while the shape of the curve remained similar.

In the experiments reported below, we present probed-sinewave stimuli monoptically and dichoptically (and binocularly) at $1.2 \mathrm{~Hz}$ and $9.4 \mathrm{~Hz}$. In the dichoptic condition, we present the probe to one 


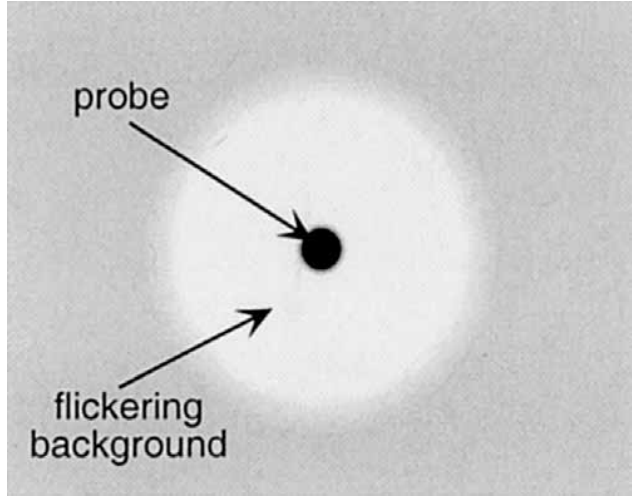

Fig. 1. Example probed-sinewave stimulus (decrement probe). The intensity of the flickering background modulates sinusoidally over time. The observer's task is to detect the probe which is presented at various times with respect to the flickering background (shown at a phase of $90^{\circ}$ in this example)

eye and the flickering background to the other eye, and in the monoptic condition we present the probe and background to the same eye (and in the binocular condition we present both the probe and the background to both eyes). The resulting fused stimulus looks like that shown in Fig. 1. This technique has been employed extensively in visual psychophysics.

The general notion behind this technique is the following: If the flickering background does not affect probe threshold in the dichoptic condition (when the probe and background are presented to different eyes) but does in the monoptic condition (when presented to the same eye), we take this as evidence that the variations in probe threshold in the monoptic condition are probably the result of retinal processing. (A caveat: Of course we are not claiming that all of the processing in the task is retinal.) If, however, the dichoptic and monoptic results are the same, the substrate for the task is probably cortical. (A further caveat: We use the term "retinal" as shorthand for "a place with primarily monocular input", and we use the term "cortical" as shorthand for "a place with primarily binocular input".)

Battersby and Wagman (1962) used this technique to investigate light adaptation. They used a background with abrupt onset

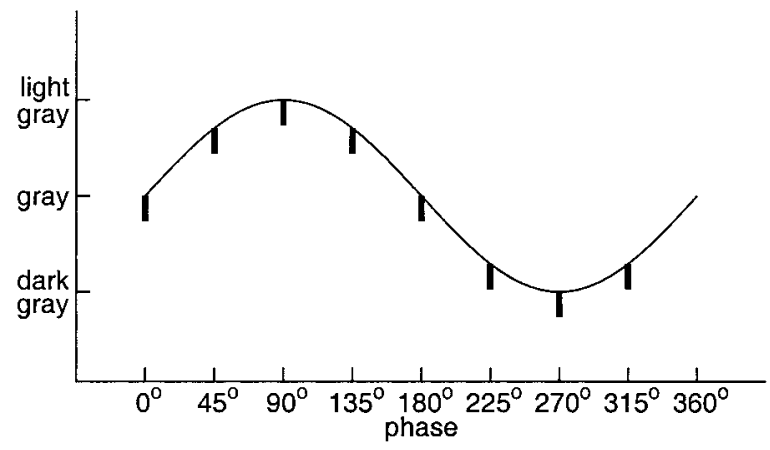

Fig. 2. The eight different phases at which the probe is presented on the flickering background in our experiments. and offset, and found only a small dichoptic effect (relative to the size of the monoptic effect). Here we extend this technique to flickering backgrounds.

Overall, our results suggest that processing in the probedsinewave task is primarily retinal. However, a slight modulation and dc-level elevation in the dichoptic results suggest some cortical contribution, particularly when the frequency of the flickering background is increased (from $1.2 \mathrm{~Hz}$ to $9.4 \mathrm{~Hz}$ ).

\section{Methods}

\section{Observers}

All observers were Columbia University undergraduates (or recent graduates). Two of the observers ( $\mathrm{AF}$ and $\mathrm{MK}$ ) were not naive as to the purpose of the present experiments. The other two observers (HH and JC) had participated in previous probed-sinewave experiments but were naive. All observers had normal or corrected-tonormal visual acuity.

\section{Apparatus}

The stimuli were presented on an AppleVision 1710 monitor ( $75 \mathrm{~Hz}$ refresh rate) controlled by an Apple Macintosh 9500. The mean luminance of the stimuli was approximately $52 \mathrm{~cd} / \mathrm{m}^{2}$. Lookup-table values were set so that the relationship between pixel value and display luminance was linear. The viewing distance was $50 \mathrm{~cm}$ for all observers. The experimental room was dimly lit.

The stimuli were viewed through a system of mirrors (see top-down view in Fig. 4) such that half of the monitor was seen only by the right eye and the other half of the monitor was seen only by the left eye. This setup enabled us to present a stimulus to the left eye (for example, the flickering background) and a different stimulus to the right eye (for example, the probe). To minimize light scatter, we used a box (with open ends) lined with black light-absorbing paper. The box was placed between the monitor and the mirrors (creating a "tunnel"). Inside the box was a divider between the two halves of the monitor. The divider, the backs of the mirrors, and the mirror holders were also covered in lightabsorbing paper. Observers used a chin rest to which each attached

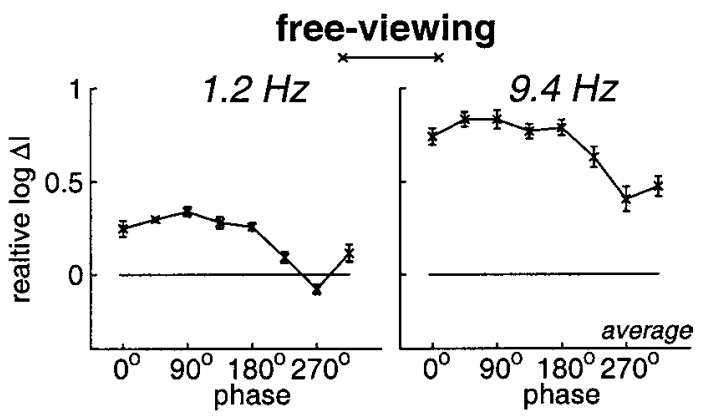

Fig. 3. Probed-sinewave data at $1.2 \mathrm{~Hz}$ and $9.4 \mathrm{~Hz}$, averaged across three observers. Probe detection threshold is plotted relative to the steady-state detection threshold (horizontal line at zero). An example of the stimulus is shown in Fig. 1. This data is from Wolfson and Graham (2000) Fig. 7 and was collected under "free-viewing" conditions (i.e. the observer did not use the apparatus shown here in Fig. 4). Error bars show \pm 1 standard error (S.E.) of the mean across observers. 


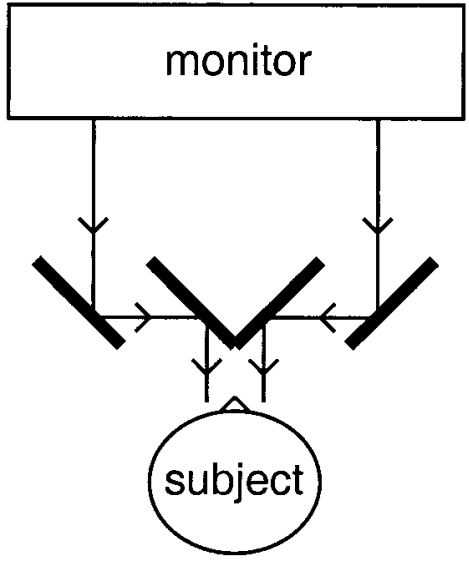

Fig. 4. Top-down sketch of the experimental setup. One half of the monitor projects to the observer's left eye and the other half of the monitor projects to the observer's right eye.

a individually tailored "mask". The mask had two small holes (approximately 2-3 $\mathrm{mm}$ ) through which the observer viewed the stimuli (such that the observer only saw the monitor's screen through the holes).

\section{Stimuli}

Stimuli were generated and presented using MathWork's MATLAB with the Psychophysics Toolbox extensions (Brainard, 1997; Pelli, 1997). There were five different stimulus layouts as shown in Fig. 5. For each of these layouts, the left side was projected to the left eye of the observer and the right side was projected to the right eye of the observer, resulting in a fused percept of one large flickering background on which the small test probe appeared. The following is a list of the viewing conditions:

- Dichoptic-Probe presented to one eye, background presented to the other eye (Fig. 5, rows $1 \& 2$ ).

- Monoptic-Probe and background both presented to one eye (Fig. 5, rows $3 \& 4$ )

- Binocular-Probe and background both presented to both eyes (Fig. 5, row 5).

The probe was 1.5 degrees of visual angle (deg) (including a smoothed edge of width $0.5 \mathrm{deg}$ ) and the flickering background was $10 \mathrm{deg}$ (including a smoothed edges of width $3 \mathrm{deg}$ ). As shown in Fig. 5, the flickering background was surrounded in black (circle diameter $12 \mathrm{deg}$ ) with four small (less than $1 \mathrm{deg}$ ) black lines pointing towards the center of the stimulus to help the observer get the images properly aligned. The frequencies of the flickering background were $1.2 \mathrm{~Hz}$ and $9.4 \mathrm{~Hz}$ (a low frequency and a frequency enough higher to elicit qualitatively different results). The probe was always a decrement in intensity with respect to the flickering background. Decrement probes were used (rather than increment probes) since, at $9.4 \mathrm{~Hz}$, the increment probe thresholds of some observers (at some phases of the flickering background) are greater than the maximum possible probe intensity (Wolfson \& Graham, 2001).

The probe was presented for one frame (which was $1 / 75$ th of a second since the monitor's refresh rate was $75 \mathrm{~Hz}$ ). The mean
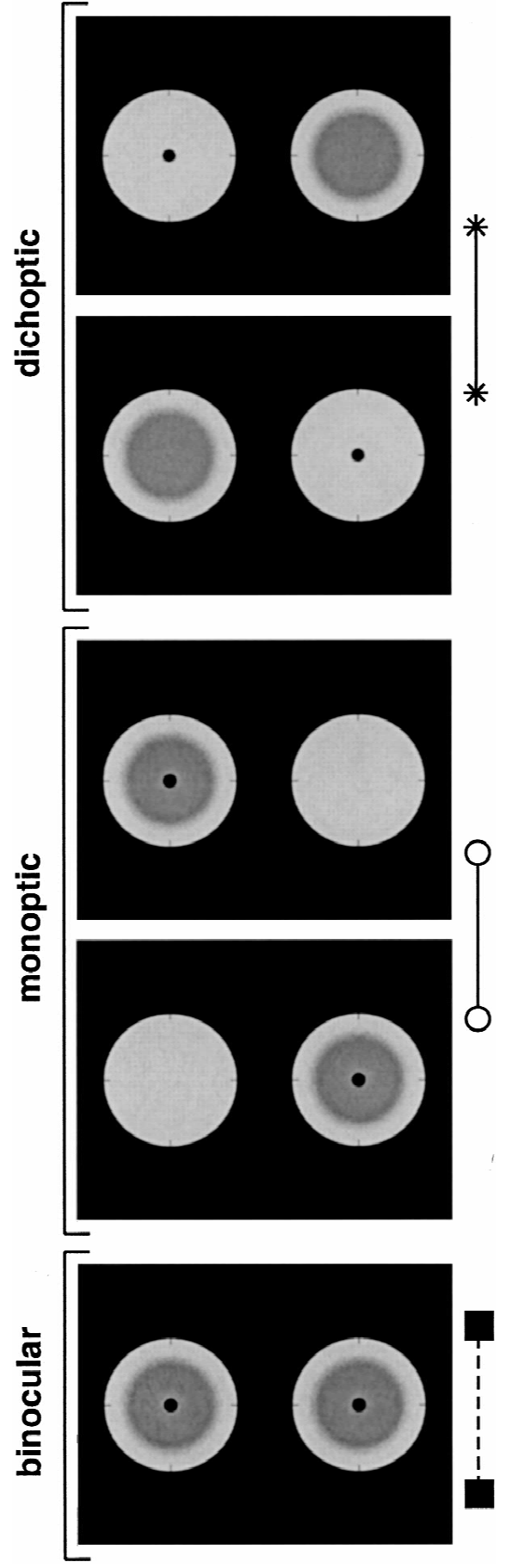

Fig. 5. The five different stimulus layouts. The top two are dichoptic (stimulate one eye with the probe and the other eye with the flickering background). The next two are monoptic (stimulate one eye with both the probe and the flickering background). The bottom is a binocular stimulus (stimulates both eyes with both the probe and the flickering background). When viewed through the system of mirrors (Fig. 4) each stimulus would fuse (be seen as one probe on one background). The phase of the flickering background in each of the layouts shown here is $270^{\circ}$. The symbols on the right of each layout are used in the data figures (Figs. 6-7 \& 9-10). 
intensity during the frame is what is reported as the probe's intensity. For a further discussion of the CRT display, see Wolfson and Graham (2001)

The flickering background's modulation was $57 \%$ in the $1.2 \mathrm{-Hz}$ conditions and $28.5 \%$ in the $9.4-\mathrm{Hz}$ conditions. We have shown (in free-viewing experiments, Wolfson \& Graham, 2001) that results are quite similar with $57 \%$ and $28.5 \%$ modulations, but the $28.5 \%$ modulation results are slightly less elevated. Because of this we used the lower modulation in the $9.4-\mathrm{Hz}$ conditions so that thresholds would not be greater than intensities that are physically realizable on the monitor (this was unnecessary in the $1.2 \mathrm{-Hz}$ conditions since the thresholds are much lower).

We can consider the flickering background's modulation in terms of the observer rather than in terms of what is presented on the monitor. If the flickering background is presented to only one eye (as in the top four rows of Fig. 5), then that eye is stimulated with modulation $M$ (where $M$ is $57 \%$ at $1.2 \mathrm{~Hz}$ and $28.5 \%$ at $9.4 \mathrm{~Hz}$ ) while the other eye is stimulated with zero modulation. If we consider some later stage in the processing (after the information from the two eyes has been combined), the resulting modulation of the sum of the stimuli in the two eyes is $(M+0) \div 2$. However, in the condition when the flickering background is presented to both eyes (bottom row of Fig. 5), the modulation at either eye is $M$, and the resulting modulation at some later stage is $(M+M) \div 2$ which is still $M$.

\section{Procedure and design}

We measured probe detection threshold using a Yes/No paradigm. At the start of each session, the observer adjusted the equipment to bring the left- and right-eye images into alignment. After aligning the images, the observer pressed a key which started the session.

Each trial consisted of the following: the background flickered for $2 \mathrm{~s}$, then the background flickered for one more cycle in which the probe was presented at one of the eight possible phases, then the background flickered for another second (the flickering in these three events was continuous). After this flickering, the screen (except for the black background shown in Fig. 5) became steady gray at the mean luminance of the stimulus. After $1 \mathrm{~s}$ of steady gray, the computer beeped, indicating that the observer could respond ("y" or " $\mathrm{n}$ " on the keyboard). The observer's response initiated the next trial. No feedback was provided.

Each time a observer ran, she or he ran two sessions (randomly ordered): one session was the experimental session and the other was a steady-state session.

In an experimental session, three probe conditions were used: in two of these probe conditions there were probes (at two of the eight possible phases); in the third probe condition there was no probe (we refer to these as blank). A staircase was run for each of these three probe conditions for each of the five stimulus layouts shown in Fig. 5. Thus, an experimental session consisted of 15 (randomly intermixed) staircases, one for each of the 3 probe conditions $\times 5$ stimulus layouts $=15$ staircases. (Note that only two of the eight possible phases were used in any single experimental session. The same phases were always run together in a session. These phase pairs were $0^{\circ}$ and $90^{\circ}$, or $45^{\circ}$ and $135^{\circ}$, or $180^{\circ}$ and $270^{\circ}$, or $225^{\circ}$ and $315^{\circ}$.)

In a steady-state session, the background did not flicker $(0-\mathrm{Hz}$ flicker). Phase is irrelevant on a steady background. Thus, there were only two possible probe conditions: one in which a probe was presented, and one in which there was no probe. Since the background is steady, only three of the five stimulus layouts in Fig. 5 are distinct (the layouts in rows 1 and 3 become identical on a steady background, as do those in rows 2 and 4). Thus, a steadystate session consisted of six (randomly intermixed) staircases, one for each of the 2 probe conditions $\times 3$ stimulus layouts $=6$ staircases.

There were 16 trials in each staircase, so an experimental session consisted of $15 \times 16=240$ trials $(1 / 3$ rd of which were blank) and a steady-state session consisted of $6 \times 16=96$ trials ( $1 / 2$ of which were blanks). The intensity of the probe on each trial (within each staircase) was determined using a QUEST procedure (Watson \& Pelli, 1983). The detection threshold was set at $60 \%$. The initial probe intensity was set to the maximum available contrast (and the initial step size was large).

Each experimental session (and corresponding steady-state session) was repeated three times for each phase pair. This results in each observer running 2880 trials per frequency of the flickering background in the experimental sessions ( 240 trials per session $\times$ 4 phase pairs $\times 3$ repeats) and 1152 trials per frequency of the flickering background in the steady-state sessions ( 96 trials per session $\times 4$ phase pairs $\times 3$ repeats).

The blank trials (in the experimental and steady-state sessions) were used to measure the false alarm rate. Overall the false alarm rate was very low-averaging about $1 \%$. There were no systematic differences across conditions although observers differed in their average false alarm rate. Note that there are only two types of blank trial in experimental sessions: the flickering background is presented to both eyes, or the flickering background is presented to one eye. (Since trials of the monoptic and dichoptic conditions were intermixed in each experimental session, we cannot measure separate false alarm rates for these two conditions.) Note also that there is only one type of blank trial in steady-state sessions.

\section{Results and discussion}

\section{Dichoptic versus monoptic}

The dichoptic and monoptic data are shown in Fig. 6 for individual observers (in different rows) and Fig. 7 averaged across observers. The two columns show data obtained with the two frequencies of the flickering background $(1.2-\mathrm{Hz}$ left column, $9.4-\mathrm{Hz}$ right column). The horizontal axis shows probe phase. The vertical axis shows probe detection threshold. In Fig. 6 the threshold is shown in luminance units $(\Delta I$ is on a scale from 0 to 1 where 1 is twice the mean luminance). In Fig. 7 the threshold is shown relative to the steady-state detection threshold. In both figures, steady-state detection thresholds are shown by horizontal lines, dichoptic thresholds by star symbols, and monoptic thresholds by circle symbols.

The 1.2-Hz dichoptic and monoptic results (Figs. $6 \&$ 7, left column) are very different from each other (compare star to circle symbols within a panel). In the monoptic condition (circle symbols) probe detection threshold varies as a function of phase, with a distinct drop at $270^{\circ}$. In the dichoptic condition (star symbols), detection threshold (at all phases) is very similar to the steady-state threshold. However, dichoptic condition results are not identical to the steady-state results (compare star symbols to the horizontal line). The dichoptic thresholds are slightly higher than the steadystate thresholds (that is, the dc-level is elevated). While this elevation is slight, it is consistent. (The average elevation, \pm 1 S.E. of the mean across observers, is $0.071 \pm 0.018 \log$ units.)

The 9.4-Hz dichoptic and monoptic results are shown in the right column of Figs. 6 and 7. In the monoptic condition (circle 


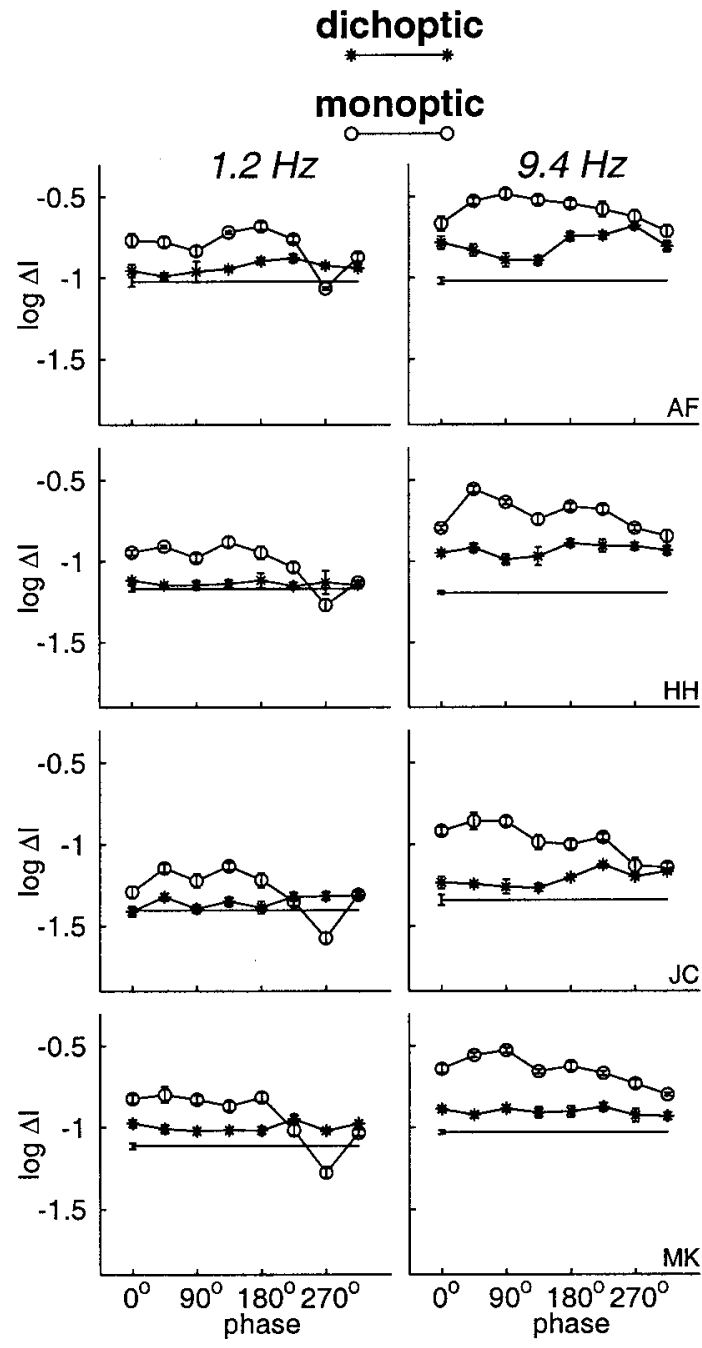

Fig. 6. Dichoptic versus monoptic probe thresholds. The dichoptic data (star symbols) is the average across the two dichoptic conditions (shown in Fig. 5, rows $1 \& 2$ ). The monoptic data (circle symbols) is the average across the two monoptic conditions (shown in Fig. 5, rows $3 \& 4$ ). The steady-state level in each panel is shown by a horizontal line. Columns show different frequencies of the flickering background; rows show different observers' data. $\Delta I$ is on a scale from 0 to 1 where 1 is twice the mean luminance. Error bars show \pm 1 S.E. of the mean across three sessions.

symbols), probe detection threshold varies as a function of phase. In the dichoptic condition (star symbols), thresholds are different from both the monoptic condition (circle symbols) thresholds and the steady-state thresholds (horizontal line). First, consider the shape of the dichoptic results. The shape-when not flat-is reversed from that in the monoptic condition. In the monoptic condition threshold is lowest when the background is dimmest $\left(225^{\circ}-315^{\circ}\right)$, but in the dichoptic condition threshold is highest during this part of the cycle. Next, consider the dc-level in the dichoptic results. The dc-level is elevated (particularly for AF and $\mathrm{HH})$ though not as elevated as in the monoptic results. (The

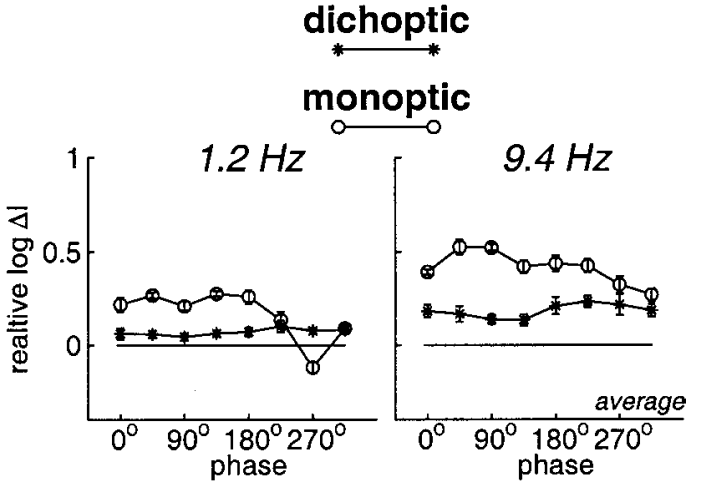

Fig. 7. Dichoptic versus monoptic probe thresholds, averaged across the four observers. The dichoptic data (star symbols) is the average across the two dichoptic conditions (shown in Fig. 5, rows $1 \& 2$ ). The monoptic data (circle symbols) is the average across the two monoptic conditions (shown in Fig. 5, rows 3 \& 4). Data are plotted relative to their steady-state levels (the steady-state level in each panel is shown by a horizontal line at 0 ). Error bars show \pm 1 S.E. of the mean across observers.

average dichoptic elevation, \pm 1 S.E. of the mean across observers, is $0.184 \pm 0.034 \log$ units; the average monoptic elevation is $0.414 \pm 0.028 \log$ units.)

All effects in the dichoptic condition that are not present in the steady-state condition should be ascribed to cortical processing. Stated another way, in the dichoptic condition the eye which views the probe does not view the flickering background, so if all the processing is retinal this condition is equivalent to the steady-state condition (in which there is no flickering background). Our results suggest that cortical processing produces

1. a small dc-elevation in the $1.2-\mathrm{Hz}$ condition,

2. a larger dc-elevation in the $9.4-\mathrm{Hz}$ condition, and

3. a small phase effect (change in shape of the threshold function) in the 9.4- $\mathrm{Hz}$ condition.

The cortical processing could be intrinsic to detecting the probe, or it could be higher level processing such as binocular rivalry. While we cannot definitively rule out higher level processes, we suspect the cortical processing is at a lower level (as in the simple model discussed in the following section).

By the simple logic presented in the Introduction, the threshold elevation in the monoptic results (both the dc-level and shape) that exceeds that in the dichoptic results is produced by retinal processing. Note that this (presumably retinal) threshold elevation is actually the majority of the threshold elevation measured here.

\section{A possible model: Part I}

Fig. 8 shows a simple schematic model that can explain the dichoptic versus monoptic results (Figs. 6 \& 7). In this model, there are three channels sensitive to the probe and thus participating in its detection. There are other channels as well but they are not shown here for simplicity's sake. The three channels differ in ocular dominance: one channel is sensitive only to the left eye (the LEFT channel), another is sensitive only to the right eye (the 


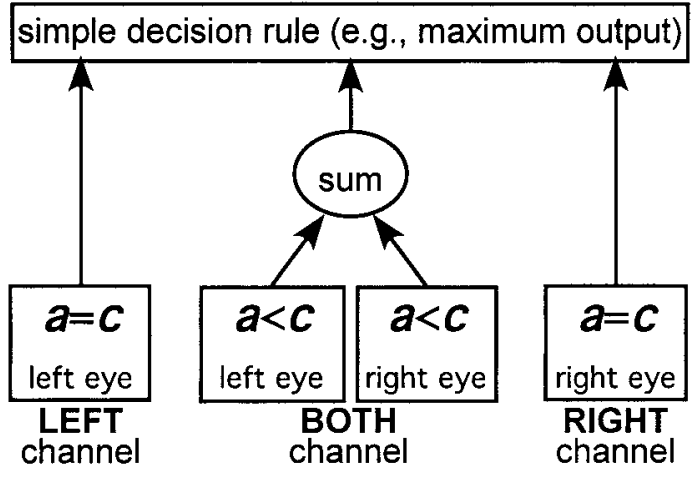

ocular dominance channels

Fig. 8. Simple schematic model. There are three ocular-dominance channels: two are monocular (LEFT and RIGHT) and one is binocular (BOTH). The amount of adaptation due to the flickering background is $a$ (where $a$ is zero if the flickering background is not viewed by that eye). Our results suggest that (1) $a$ is less in the binocular channel $(a<$ c) than in the monocular channels $(a=c)$, and (2) $c$ is greater when the background flickers at $9.4 \mathrm{~Hz}$ than at $1.2 \mathrm{~Hz}$. Note that the contrast of the flickering background used in the experiments at $9.4 \mathrm{~Hz}$ was half that used at $1.2 \mathrm{~Hz}$; thus, the adapting power for $9.4 \mathrm{~Hz}$ versus $1.2 \mathrm{~Hz}$ at identical contrasts would probably be even greater. Note also that the time course of the adaptation in the model is assumed to be such that it can produce facilitation rather than inhibition relative to the steady-state at some phases of the flickering background.

RIGHT channel), and the third channel linearly sums the activity from both eyes (the BOTH channel). The observer's response is assumed to be determined by some simple decision rule combining the outputs of these three channels (for example, the observer detects a stimulus if and only if the maximum of the channels' outputs exceeds criterion). This kind of model - in which there are multiple ocular-dominance channels - has been proposed before for psychophysical results (for example, Anderson \& Movshon, 1989). Also, single neurons in the visual cortex are well known to differ in ocular dominance (for example, Hubel \& Wiesel, 1977).

The flickering background is assumed to primarily adapt the LEFT and RIGHT channels since the measured adaptation effects in the monoptic conditions (circle symbols, Figs. $6 \& 7$ ) are much bigger than in the dichoptic conditions (star symbols). To explain the difference between the $9.4-\mathrm{Hz}$ and $1.2-\mathrm{Hz}$ monoptic conditions (which is even bigger than the difference between the $9.4-\mathrm{Hz}$ and $1.2-\mathrm{Hz}$ dichoptic conditions), one also needs to assume that adaptation of the LEFT and RIGHT channels is greater when the background is flickering at $9.4 \mathrm{~Hz}$ than at $1.2 \mathrm{~Hz}$. The flickering background is also assumed to have a small adaptation effect on the BOTH channel (in all conditions). To explain the dichoptic results, this adaptation effect on the BOTH channel must be greater when the background is flickering at $9.4 \mathrm{~Hz}$ than at $1.2 \mathrm{~Hz}$. In summary, we assume that

1. adaptation effects on the LEFT, RIGHT, and BOTH channels are greater at $9.4 \mathrm{~Hz}$ than at $1.2 \mathrm{~Hz}$, and

2. adaptation effects on the LEFT and RIGHT channels are equal (to one another) and greater than the adaptation effects on the BOTH channel. We will return to this model after presenting the rest of the experimental data.
Binocular summation?

We have not yet looked at the results from the binocular condition. Figs. 9 and 10 show the binocular results along with the monoptic results. In the binocular condition, the probe and flickering background were both presented to both eyes. In the monoptic condition, the probe and flickering background were both presented to only one eye.

The comparison between our binocular and monoptic conditions is what is often called a binocular summation experiment. An improvement of about $0.15 \log$ units (a factor of about 1.4 or $2^{1 / 2}$ ) for binocular relative to monoptic thresholds has been found in

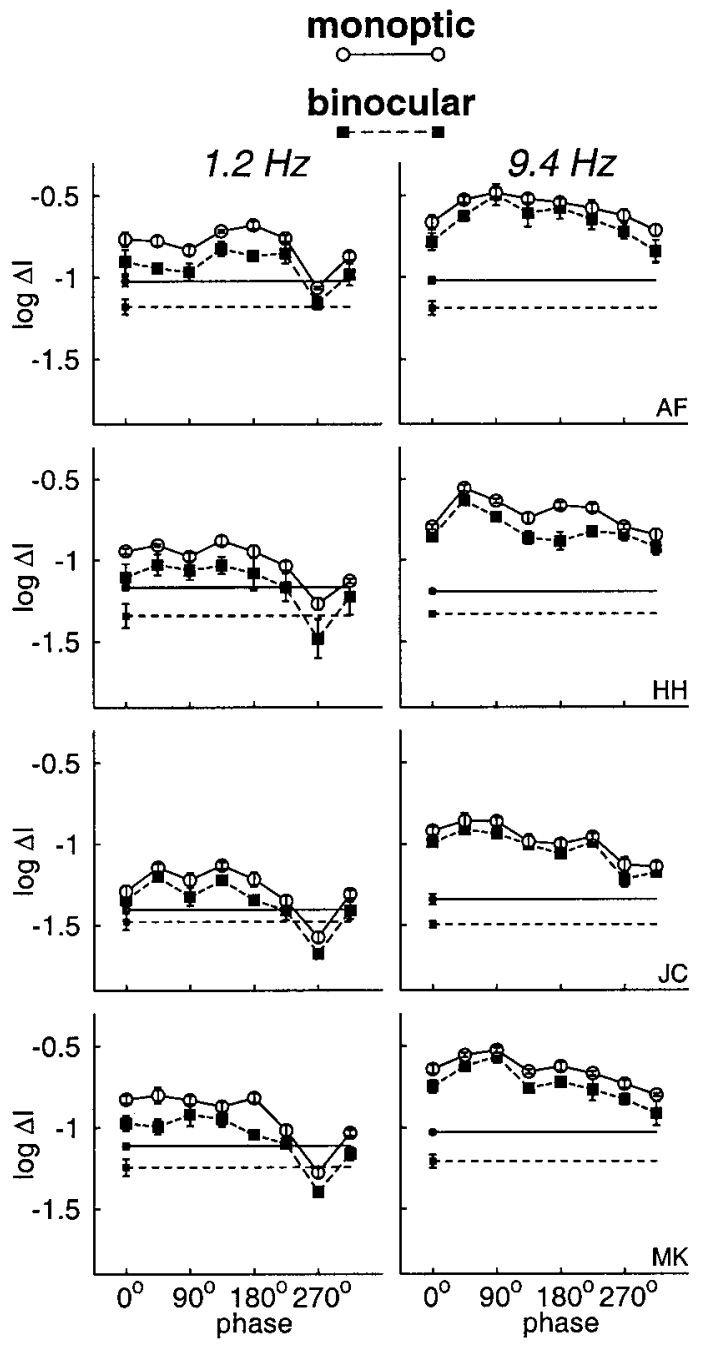

Fig. 9. Monoptic versus binocular probe thresholds. The monoptic data (circle symbols) is the average across the two monoptic conditions (shown in Fig. 5, rows $3 \& 4$ ). The binocular data is plotted with square symbols and dashed line style (the stimulus layout is shown in Fig. 5, row 5). The associated steady-state levels are shown by horizontal lines (with the same-but much smaller-symbols). Columns show different frequencies of the flickering background; rows show different observers' data. Error bars show \pm 1 S.E. of the mean across three sessions. 


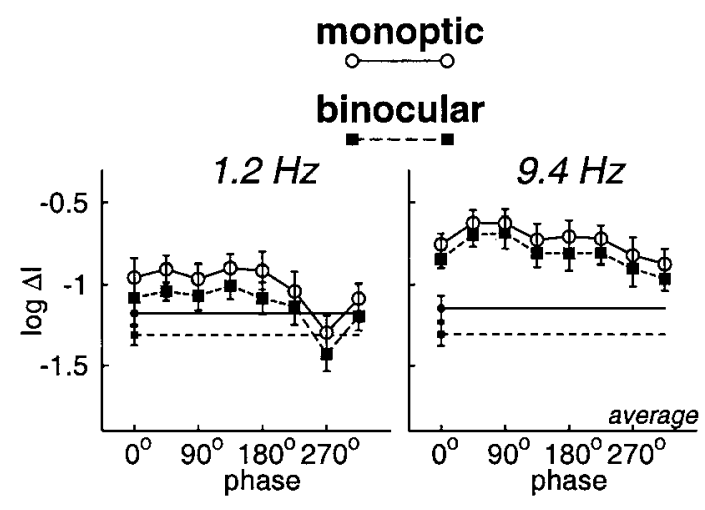

Fig. 10. Monoptic versus binocular probe thresholds, averaged across the four observers. The monoptic data (circle symbols) is the average across the two monoptic conditions (shown in Fig. 5, rows $3 \& 4$ ). The binocular data is plotted with square symbols and dashed line style (the stimulus layout is shown in Fig. 5, row 5). The associated steady-state levels are shown by horizontal lines (with the same-but much smaller-symbols). Error bars show \pm 1 S.E. of the mean across observers.

many-but not all-binocular summation experiments (see review in Graham, 1989, Chapter 12). We find that the binocular steadystate thresholds (dashed horizontal lines, Figs. $9 \& 10$ ) are approximately $0.15 \log$ units lower than the monoptic steady-state thresholds (solid horizontal lines). The mean difference across all sessions ( \pm 1 S.E. of the mean across observers) is $0.148 \pm 0.012$ $\log$ units (which is $0.135 \pm 0.022 \log$ units in the $1.2-\mathrm{Hz}$ sessions and $0.160 \pm 0.009 \log$ units in the $9.4-\mathrm{Hz}$ sessions).

Now consider the probe thresholds on the flickering background in Figs. 9 and 10. When the background flickers at $1.2 \mathrm{~Hz}$, the difference between the binocular (square symbols) and monoptic (circle symbols) probe thresholds is $0.121 \pm 0.012 \log$ units, which is very like - though slightly smaller than - the binocular summation in the steady-state conditions $(0.148 \pm 0.012 \log$ units $)$. The difference at $9.4 \mathrm{~Hz}$ is definitely smaller $(0.083 \pm 0.011 \mathrm{log}$ units).

\section{A possible model: Part II}

Why should there be a difference of about $0.15 \log$ units (approximately the difference between the binocular and monoptic steadystate thresholds here) for binocular summation in any condition? A somewhat smaller effect is expected from so-called probability summation of independent channels (approximately $0.08 \mathrm{log}$ units or a factor of about $2^{1 / 4}$ ), and this smaller factor is what is found for summation across two far-apart values on many other dimensions (for example, spatial frequency, spatial position, orientation; see review in Graham, 1989, Chapters 4 \& 12). The existence of the BOTH channel in the simple model of Fig. 8 is one explanation of the greater factor found for binocular summation. The predicted amount of summation over the LEFT, RIGHT, and BOTH channel outputs is greater than that expected from only the LEFT and RIGHT channel outputs.

Why do we find less binocular summation in the $9.4-\mathrm{Hz}$ condition than in the $1.2-\mathrm{Hz}$ condition (and less in the $1.2-\mathrm{Hz}$ condition than in the steady-state condition)? These differences can be predicted by the assumption in the simple model of Fig. 8- previously used to explain the results in the dichoptic conditionthat the BOTH channel is adapted more by the $9.4-\mathrm{Hz}$ flickering background than by the $1.2-\mathrm{Hz}$ background. Also, as one changes from a monoptic to a binocular stimulus, there is a greater contribution of the BOTH channel to detection. Thus, in the $1.2-\mathrm{Hz}$ binocular condition the BOTH channel is less adapted (than at $9.4 \mathrm{~Hz}$ ) and more influential (than in the monoptic condition), resulting in greater binocular summation at $1.2 \mathrm{~Hz}$ than at $9.4 \mathrm{~Hz}$. Similar logic applies when comparing results on the $1.2 \mathrm{-Hz}$ flickering background to those on a steady background.

Thus, the simple model in Fig. 8 is consistent with our results.

\section{Implications for dynamic models of light adaptation}

These results have some implications for the plausibility of mechanisms used in current dynamic models of light adaptation. The models which have predicted some probed-sinewave results successfully are those of Wilson (1997) and Snippe et al. (2000).

The success of Wilson's (1997) model depends on a push-pull mechanism (Hood \& Graham, 1998; Wolfson \& Graham, 2000, 2001). The available evidence suggests that there is no push-pull mechanism in the retina (Hood, 1998). Here we find evidence that most of the probed-sinewave effects are likely retinal.

Snippe et al.'s (2000) model depends critically on a contrast gain control. There is evidence that such contrast gain control exists in the retina (see Hood, 1998, for references).

\section{Conclusion}

Our results suggest that most of the processing in the probedsinewave paradigm is retinal (or any place with primarily monocular input). However, there is a small cortical (or any place with primarily binocular input) contribution, particularly at higher frequencies of the flickering background $(9.4 \mathrm{~Hz}$ compared to $1.2 \mathrm{~Hz}$ here).

\section{Acknowledgments}

We would like to thank our observers for their many hours of work. This research was supported by National Eye Institute grants EY08459 to Norma Graham and EY06933 to Sabina Wolfson, and by summer undergraduate research fellowships from Columbia University to Arusha Farahani and Michael Krug in the summer of 2000. Portions of this work were presented at ARVO (Wolfson et al., 2001)

\section{References}

Adelson, E.H. (1982). Saturation and adaptation in the rod system. Vision Research 22, 1299-1312.

Anderson, P.A. \& Movshon, J.A. (1989). Binocular combination of contrast signals. Vision Research 29, 1115-1132.

Battersby, W.S. \& Wagman, I.H. (1962). Neural limitations of visual excitability. IV: Spatial determinants of retrochiasmal interaction. American Journal of Physiology 203, 359-365.

Boynton, R.M., Sturr, J.F. \& Ikeda, M. (1961). Study of flicker by increment threshold technique. Journal of the Optical Society of America 51, 196-201.

Brainard, D.H. (1997). The psychophysics toolbox. Spatial Vision 10, 443-446.

CrawFord, B.H. (1947). Visual adaptation in relation to brief conditioning stimuli. Proceedings of the Royal Society B 134, 283-302.

DE LANGE, H. (1958). Research into the dynamic nature of the human fovea-cortex systems with intermittent and modulated light. Journal of the Optical Society of America A 7, 2223-2237.

DeMarco, P.J., Hughes, A. \& Purkiss, T.J. (2000). Increment and decrement detection on temporally modulated fields. Vision Research 40, 1907-1919. 
Geisler, W.S. (1978). Adaptation, afterimage and cone saturation. Vision Research 18, 279-289.

Graham, N. (1989). Visual Pattern Analyzers. New York: Oxford University Press.

Graham, N. \& Hood, D.C. (1992). Modeling the dynamics of light adaptation: The merging of two traditions. Vision Research 32, 1373-1393.

Hood, D.C. (1978). Psychophysical and electrophysiological tests of physiological proposals of light adaptation. In Visual Psychophysics: Its Physiological Basis, ed. Armington, J., Krauskopf, J. \& Wooten, B. pp. 141-155. New York: Academic Press.

HooD, D.C. (1998). Lower-level visual processing and models of light adaptation. Annual Review of Psychology 49, 503-535.

Hood, D. C. \& Finkelstein, M. A. (1986). Sensitivity to light. In Visual Psychophysics: Its Physiological Basis, ed. Boff, K.R., Kaufman, L. \& Thomas, J.P. Chapter 5. New York: Academic Press.

Hood, D.C. \& Graham, N. (1998). Threshold fluctuations on temporally modulated backgrounds: A possible physiological explanation based upon a recent computational model. Visual Neuroscience 15, 957-967.

Hood, D.C., Graham, N., von Wiegand, T.E. \& Chase, V.M. (1997) Probed-sinewave paradigm: A test of models of light-adaptation dynamics. Vision Research 37, 1177-1191.

Hubel, D.H. \& Wiesel, T.N. (1977). Functional architecture of macaque monkey visual cortex. Proceedings of the Royal Society B (London) 198, $1-59$.

KelLy, D.H. (1961). Visual responses to time-dependent stimuli: II. Single channel model of the photopic visual system. Journal of the Optical Society of America 51, 747-754.

Maruyama, K. \& TAKAHASHI, M. (1977). Wave form of flickering stimulus and visual masking function. Tohoku Psychologica Folia 36, $120-133$.
Pelli, D.G. (1997). The videotoolbox software for visual psychophysics: Transforming numbers into movies. Spatial Vision 10, 437-442.

Shady, S. (1999). Comparing the dynamic mechanisms of light adaptation of the rod and cone systems: Empirical investigation and theoretical analysis. Doctoral Dissertation, Columbia University, New York, NY.

Shapley, R. \& Enroth-Cugell, C. (1984). Visual adaptation and retinal gain controls. In Progress in Retinal Research, ed. Osborne, N.N. \& Chader, G.J., pp. 263-343. Oxford: Pergamon Press.

Shickman, G.M. (1970). Visual masking by low-frequency sinusoidally modulated light. Journal of the Optical Society of America 60, 107117.

Snippe, H.P., Poot, L. \& van Hateren, J.H. (2000). A temporal model for early vision that explains detection thresholds for light pulses on flickering backgrounds. Visual Neuroscience 17, 449-462.

von Wiegand, T.E., Hood, D.C. \& Graham, N. (1995). Testing a computational model of light-adaptation dynamics. Vision Research $\mathbf{3 5}$, 3037-3051.

WATSON, A.B \& Pelli, D.G (1983). QUEST: A Bayesian adaptive psychometric method. Perception and Psychophysics 33, 113-120.

WiLson, H.R. (1997). A neural model of foveal light adaptation and afterimage formation. Visual Neuroscience 14, 403-423.

Wolfson, S.S. \& GRAham, N. (2000). Exploring the dynamics of light adaptation: The effects of varying the flickering background's duration in the probed-sinewave paradigm. Vision Research 40, 2277-2289.

Wolfson, S.S. \& Graham, N. (2001). Comparing increment and decrement probes in the probed-sinewave paradigm. Vision Research $\mathbf{4 1}$, 1119-1131.

Wolfson, S.S., Graham, N., Krug, M. \& Farahani, A. (2001). Monoptic versus dichoptic presentation in the probed-sinewave paradigm. Investigative Ophthalmology and Visual Science 42 (Abstract \#841), S157. 